\title{
DYKE-DAVIDOFF-MASSON SYNDROME PRESENTING WITH RECURRENT SEIZURES
}

\author{
M. Rajaguru', V. Umamaheswara Reddy ${ }^{\mathbf{2}}$ Amit Agrawal $^{\mathbf{3}}$, V. Ganesh ${ }^{\mathbf{1}}$, Anil Tadikonda ${ }^{\mathbf{1}}$ \\ ${ }^{1}$ Department of Neurology, Narayana Medical College Hospital, Chinthareddypalem, Nellore, \\ Andhra Pradesh, India \\ ${ }^{2}$ Department of Radiology, Narayana Medical College Hospital, Chinthareddypalem, Nellore, \\ Andhra Pradesh, India \\ ${ }^{3}$ Department of Neurosurgery, Narayana Medical College Hospital, Chinthareddypalem, Nellore, \\ Andhra Pradesh, India
}

\begin{abstract}
Dyke-Davidoff-Masson syndrome (DDMS) is cerebral hemiatrophy occurring following brain insult resulting from infarct, trauma or infection in utero or soon after birth. Clinical features of this syndrome include variable degree of contralateral hemiparesis, facial asymmetry, seizures and mental retardation. Recurrent seizures is the most debilitating and poor prognostic indicator of this disease. Neuroimaging plays pivotal role in establishing the diagnosis of DDMS syndrome. CT/MR imaging shows unilateral cerebral parenchymal atrophy, prominent sulcal spaces, ipsilateral ventriculomegaly, cerebellar atrophy, falcine and superior Sagittal sinus shift to affected side. Bone windowing in CT scan shows decreased skull volume on affected side calvarial thickening, elevation of sphenoid wing and petrous temporal bone, expanded sinuses and mastoids. Here we describe 34 years old female who had hemiparesis, seizures and facial asymmetry since childhood and imaging evaluation established diagnosis of congenital type of DDMS.
\end{abstract}

Keywords: Dyke-Davidoff-Masson syndrome, seizures, hemiatrophy and ventriculomegaly

\section{INTRODUCTION}

Dyke-Davidoff-Masson syndrome (DDMS) is cerebral hemiatrophy occurring following insult resulting from infarct, trauma or infection in utero or soon after birth. (1-4) Clinical features of this syndrome include variable degree of contralateral hemiparesis, facial asymmetry seizures and mental retardation. $(3,5)$ Imaging of brain will reveal diffuse volume loss of brain on the affected side, prominent sulcal spaces and dilated ventricle on the atrophic side and sometimes gliosis (2-5). Inter hemispheric fissure and sagittal sinus are displaced beyond midline to the affected side. Bone windowing in CT scan shows decreased skull volume on affected side, calvarial thickening, elevation of sphenoid wing and petrous temporal bone, expanded sinuses and mas- toids. $(1,6)$ MRI provides similar detail and also helps differentiating other radiological mimics. Here we describe 34 years old female who had hemiparesis, seizures and facial asymmetry since child hood and imaging evaluation established diagnosis of congenital type of DDMS.

\section{CASE REPORT}

34 years young lady came with complaints of recurrent seizure episodes since 4 years of her age. Her seizures were left focal getting secondarily generalised. She had received antiepileptic drugs T. Primidone $250 \mathrm{mg} 1 \mathrm{HS}$ for 30 years and T. Epilex chrono $300 \mathrm{mg}$ one tab three times/day for 10 years. Inspite of good drug compliance her seizures haven't stopped. She gets seizures once a day least 


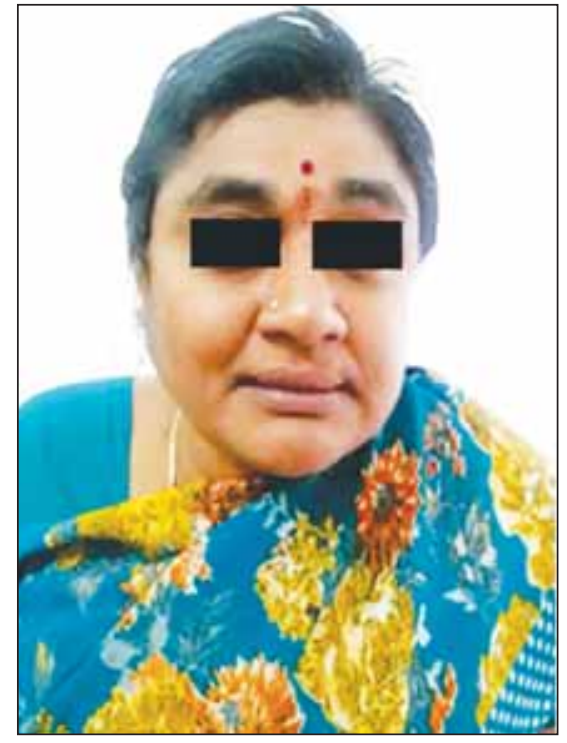

FIGURE 1. Clinical photograph of the patient showing facial asymmetry

3-4 days in a week, last episode of seizure on the day when she came to this hospital on 12.2.15. She had headache also for the past 2 months. She is married but no children. Her menstrual history is normal. She studied up to fifth standard and her scholastic was performance poor. Neurological examination revealed mental retardation of MMSE score 15 . She had dystonic involuntary movement is her left hand \& left foot. She had spastic weakness of left upper and lower limb 4/5, deep tendon jerks were normal right side and brisk on the left side, she also had facial asymmetry. Her plantars response was down going on both side. MRI brain revealed atrophic cerebral hemisphere on the right side with hypoplasia of skull vault with prominent frontal and ethmoidal sinuses and overriding of falx cerebri on the right side, cerebellar atrophy (Fig. 2, 3). EEG showing seizure activity on the right side. The MMSE score was 15 and her lobar function revealed following abnormality impaired attention, deficient found of information and impaired abstract thinking, delayed recall, impaired remote memory. Based on the history, clinical findings, MRI brain features and EEG we diagnosed this is a case of Dyke-DavidoffMasson syndrome. As seizures were not controlled on existing medication extended anticonvulsant therapy with newer antiepileptics drugs was prescribed and was suggested follow up. First monthly follow up was done but there were no signs decrease frequency of seizures attacks.

\section{DISCUSSION}

DDMS was described by Dyke-Davidoff and Masson in their study of skull radiographs and

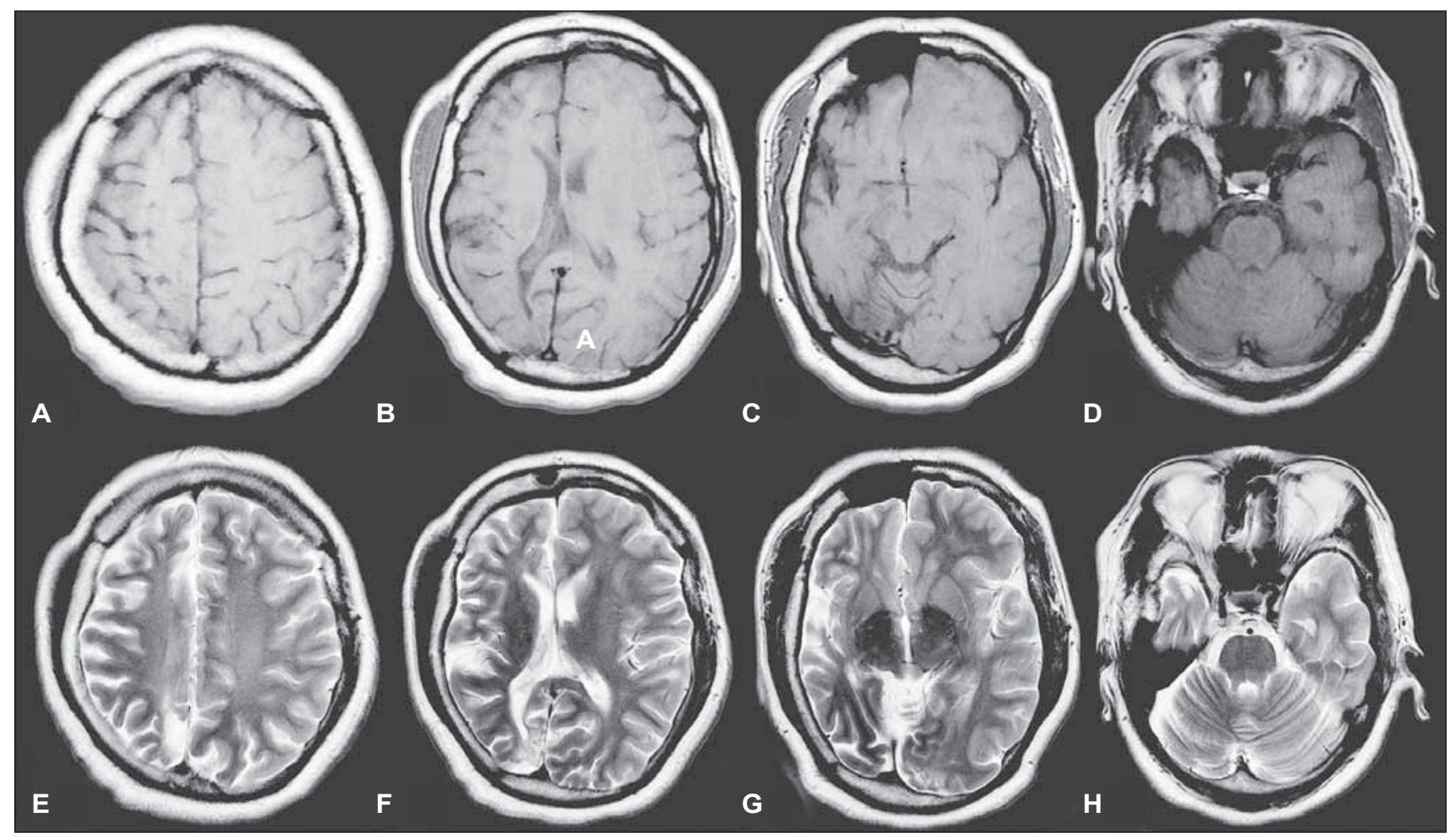

FIGURE 2. Axial T1, T2 images (A-F) showing right sided hemiatrophy with prominent sulcal spaces, ipsilateral lateral ventriculomegaly, paranasal sinuses and mastoid hypertrophy. Mild cerebellar atrophy and shift of falx to same side is also seen. 


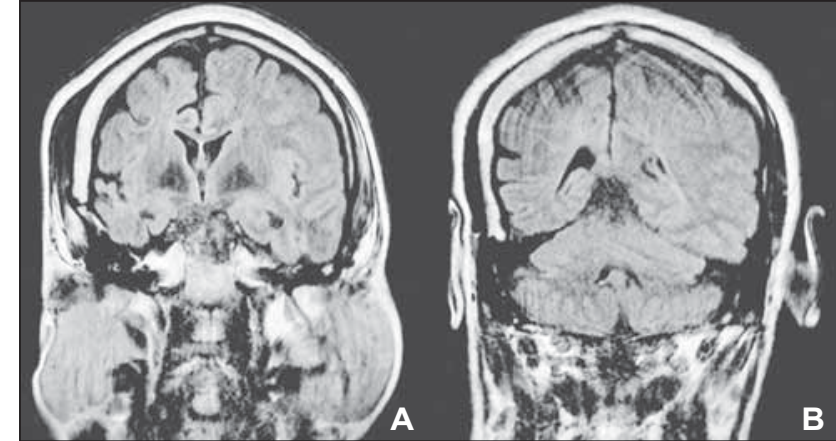

FIGURE 3. FLAIR coronal images showing right cerebral atrophy and calavarial thickening $(A, B)$

pneumoencephalographic data in 9 patients whose characteristics included hemiparesis, seizures, facial-asymmetry, and mental retardation in 1933. (2) Hangmen etal in their study of 19 cases showed that males are more often affected than females by DDMS and left sided hemiatrophy is more common than right sided hemiatrophy (6). Etiologic factors responsible for cerebral hemiatrophy can be classified into: congenital or acquired. $(1-3,5,6)$ Congenital hemiatrophy probably occurs mainly due vascular causes (Middle cerebral artery occlusion) during intrauterine life. $(2,5,7)$ Acquired hemiatrophy is generally due to many inciting factors occurring during perinatal period like infection, trauma, ischaemic and haemorrhagic lesions. $(1,2,5,7)$ Coarctation of the midaortic arch also has been described as a rare cause of DDMS. Congenital hemiatrophy with prominent sulcal spaces on affected side, ipsilateral midline shift and gliotic tissue is generally absent in this type. (2) Consistent clinical picture of DDMS includes hemipariesis, facial asymmetry and seizures. Mental retardation may be present at the time of clinical presentation or may occur years or months after hemipariesis has occured. $(2,3)$. Depending on degree and extent of brain involvement on affected side sensory, speech and psychiatric disturbances may also occur. $(5,6,8)$ In our case along with consistent clinical picture, patient also had dystonia in left upper limb and foot. Neuroimaging plays a pivotal role in establishing the diagnosis of this syndrome. Computed tomography reveals unilateral cerebral parenchymal atrophy, prominent sulcal spaces, ipsilateral ventriculomegaly, cerebellar atrophy, falcine and sagittal sinus shift to the affected side. Skull changes are adaptation to compensate the volume loss of neural tissue. (1) Brain reaches three-fourths of its adult size by three years and shape of the skull is influenced by outward pressure exerted by growing brain parenchyma. When the brain growth is shunted by various factors, there will be pressure in opposite direction resulting in expansion of the paranasal sinuses, mastoids and widening of diploic spaces, elevation of orbital, sphenoid wing and petrous ridges with decrease in size of anterior and middle cranial fossa. (2) Differential diagnosis for DDMS are Sturge-Weber syndrome, Rasmussen encephalitis, brain tumors (germinoma), Parry Romberg syndrome, linear nevus sebaceous syndrome. A proper clinical history and multisystem examination will provide the correct diagnosis in most of the cases. $(1,2,6,8)$ Treatment of DDMS is mainly symptomatic with anticonvulsive therapy. Neurorehabilatation for hemipariesis and learning disabilities can be advised. Hemispherectomy is the surgical treatment option in carefully selected group of patients. Recurrent seizures and early onset hemiparesis are poor prognostic indicators for this condition. (1)

\section{CONCLUSION}

DDMS is a rare cause of recurrent seizures. Clinical features are quite variable in this condition earlier many authors have described sensory, speech and psychiatric (schizophrenia) in association with consistent clinical picture of hemipariesis, seizures and mental retardation, however as for our knowledge this is the first case report on DDMS syndrome in which patient presented to us with dystonia. Proper clinical history, multisystem examination and appropriate neuroimaging are required for correct diagnosis and in differentiating other causes of hemiatrophy. Recurrent seizures and early onset hemiparesis are poor prognostic indicators for this condition. 


\section{REFERENCES}

1. Aguiar P.H., Ching W.I., Leitão H., et al. MR and CT imaging in the Dyke-Davidoff-Masson syndrome: report of three cases and contribution to pathogenesis and differential diagnosis. Arquivos de neuro-psiquiatria 1998; 56:803-807.

2. Sharma S., Goyal D., Negi A., Sood R., Jhobta A., Surya M. Dyke-davidoff masson syndrome. Indian Journal of Radiology and Imaging 2006; 16:165.

3. Shetty D., Lakhkar B., John J. Dyke-Davidoff-Masson syndrome. Neurology India 2003; 51:136.

4. Verma R., Sahu R. Dyke-Davidoff-Masson syndrome. BMJ case reports 2012; 2012:bcr2012006729.

5. Goyal J., Shah V., Rao S., Jindal N. Dyke Davidoff Masson syndrome in Children. The Internet Journal of Pediatrics and Neonatology 2009; 10:101-107.
6. Rath B., Samant M., Swain K., Mallick A. Dyke-Davidoff-Masson syndrome: A case report. Medical Journal of Dr DY Patil University 2014; 7:672.

7. Singh P., Saggar K., Ahluwalia A. Dyke-Davidoff-Masson syndrome: classical imaging findings. Journal of pediatric neurosciences 2010; 5:124.

8. Jain D., Aggarwal H.K., Goyal S., Mittal A. Dyke-Davidoff-Masson syndrome: A rare case report. Iranian Journal of Neurology 2014; 13:255-256. 